\title{
WISNIA-WEILL, V. LES NOUVEAUX POUVOIRS D'AGIR. 112 PÁGS. PARÍS, 2020: SEUIL
}

\author{
Prof. Eguzki Urteaga ${ }^{1}$ \\ Universidad del País Vasco
}

Vanessa Wisnia-Weill acaba de publicar su libro, titulado Les nouveaux pouvoirs d'agir, en la colección La République des Idées de la editorial Seuil. Conviene recordar que la autora es diplomada de las prestigiosas Escuela Politécnica y Escuela Nacional de Puentes y Calzadas. Tras trabajar durante varios años en la banca de negocios, se incorpora a los servicios del Primer Ministro galo. De 2009 a 2017, forma parte del Departamento de Cuestiones sociales de Francia Estrategia, como experta de las cuestiones educativas y sociales ( La vie des idées, 2019). Ha publicado numerosos informes y notas de análisis titulados Augmenter aussi le nombre de bons élèves (2014) o Aménagements souples du temps et du lieu de travail, que forma parte del informe Disposer de temps et de droits pour s'occuper de ses enfants, de sa famille et de ses proches en perte d'autonomie (2018). Junto con Cécile Jolly y Emmanuelle Prouet, ha redactado los informes Repenser la protection des actifs (2016) y Comment mieux protéger les actifs du risque de perte ou d'insuffisance de revenus? (2017), y, con François de Singly, ha escrito Pour un développement complet de l'enfant et de l'adolescent (2015). Es actualmente Secretaria General adjunta del Alto Consejo de la familia, de la infancia y de la edad.

En la introducción de la presente obra, la autora constata que las sociedades contemporáneas "erigen en ideal la capacidad de actuar, figura del individuo libre y autónomo" (p.7). No en vano, estas mismas sociedades muestran "exasperaciones, reivindicaciones o sufrimientos, vidas [obstaculizadas que se hallan a años luz] de ese ideal de [realización personal]. Se trata de las madres aisladas, afectadas en su [actividad] parental, [cuando] no consiguen construir un entorno satisfactorio para sus hijos, cuando el otro [progenitor] no se implica suficientemente [en la educación de los hijos], cuando las plazas de guardería para [las personas que trabajan con] horarios atípicos [escasean], cuando una movilidad residencial obligada las aleja de los abuelos ayudantes y de los servicios de proximidad" (p.7). Asimismo, para al menos un tercio de la población, los gastos obligatorios dificultan las maneras personales de socializarse, como pueden ser preparar una comida para sus amigos, compartir actividades de ocio, etc. (p. 7).

\footnotetext{
${ }^{1}$ Departamento de Sociología y Trabajo Social, Facultad de Relaciones Laborales y Trabajo Social. E-mail: eguzki.urteaga@ehu.eus
} 
Para Wisnia-Weill, el futuro pasa por unas nuevas capacidades de actuar en varios ámbitos. "Es que las cuestiones sociales, (...) tributarias de las desigualdades socioeconómicas, no pueden hacer el impasse sobre la interpretación multidimensional de la libertad" (p. 8); sabiendo que las transiciones económicas, tecnológicas y climáticas afectan estas nuevas capacidades de actuar (p. 8). En este contexto, estima la autora, es preciso "garantizar nuestra [capacidad de actuar]. Es la razón por la cual diferentes movimientos filosóficos y políticos, en particular la teoría de las capacidades, han sentido la necesidad de [identificar] las grandes dimensiones de la existencia y de la acción humana" (p. 8). Fundamentalmente, cinco esferas moldean nuestras maneras de actuar: "el trabajo proveedor de rentas, cargos y honores; el consumo y el acceso a las necesidades básicas, especialmente en materia de sanidad, educación y seguridad; el compromiso en las relaciones personales y familiares; el conocimiento; y, la participación ciudadana" (p. 8). En este libro, muestra "lo que constituye [la capacidad de actuar] en cada esfera y en qué es un análisis pertinente para comprender la sociedad" (p. 9). Al respecto, se pregunta: “¿Dónde están los márgenes de maniobra de los individuos?” ¿Qué es problemático para que una capacidad de actuar exista en beneficio de cada uno? La respuesta a estas preguntas conduce Wisnia-Weill a poner de manifiesto los conflictos normativos en cuanto al reparto de los bienes sociales (p. 9).

En el primer capítulo, titulado "las cinco esferas de la capacidad de actuar", la autora recuerda que unos intensos debates dan cuenta de la centralidad de la figura del individuo libre y autónomo, "que se trate de aquellos que [aluden] al ideal meritocrático, que anima el individuo a [desplegarse] en la medida de lo posible, o a la concesión de [ayudas] públicas" que permiten emanciparse de ciertos determinismos sociales (p. 11).

De hecho, la libertad extrae su fuerza de la capacidad para establecer un vínculo sistémico entre el sujeto individual y el orden social. Por lo cual, "su garantía es indisociable del (...) pluralismo democrático" (p. 11). Esta lógica desemboca en el derecho a gozar de libertades negativas, tales como la libertad de conciencia y las libertades civiles (p. 12). A pesar de ser ineludible, esta concepción no toma suficientemente en consideración las condiciones de vida de las personas (p. 12). Por consiguiente, es preciso añadirles, por una parte, la libertad reflexiva a través de la cual "el individuo de refiere a sí mismo y a sus actos", y, por otra parte, la libertad social que "intenta pensar las condiciones de realización de los objetivos de vida" (p. 12). La perspectiva elegida por la autora aspira a "garantizar a todos los ciudadanos unas libertades efectivas para actuar y llevar a cabo una vida de manera singular, teniendo en cuenta unas instituciones en las cuales evalúan las acciones y las interdependencias sociales y relacionales" (p. 13). 
Más concretamente, "la profundización de un individualismo de la singularidad [implica elaborar] una teoría coherente de la justicia social"' (p. 13). La perspectiva de las capacidades ofrece una respuesta adecuada al respecto, dado que "la sociedad de las capacidades garantiza que cada uno pueda desarrollar unas capacidades internas en varios [ámbitos], pero también se encuentra en unas condiciones de vida que permiten efectivamente, como mínimo, unas realizaciones [personales], a través de unas combinaciones singulares de sus facultades. (...) Es un mínimo que puede [resultar] muy exigente" (p. 15).

La cuestión del umbral mínimo es susceptible de cambiar "el alcance político de una capacidad" (p. 16). Por sí sola, "no permite [alcanzar] un objetivo universal y adaptarlo a un nivel de desarrollo [personal]" (p. 16). La toma en consideración de las realidades sociales "conduce rápidamente a considerar los determinantes del reparto de bienes en un país" (p. 16). En las sociedades democráticas, Walzer (1983) identifica tres principios legítimos de distribución que valen de manera preferencial en ciertas esferas: "satisfacción incondicional de las necesidades básicas, equidad en la meritocracia, lucha contra las desigualdades en el seno de un sistema de intercambios mercantiles" (p. 17). A las realidades sociales se añaden las realidades físicas que determinan notablemente nuestras acciones. Así, el periodo de transición climática influye en nuestras condiciones de vida (p. 18).

La autora se centra en la capacidad de actuar del individuo, "en sus márgenes de maniobra, tal como participan al sentimiento de existencia, a la sensación de empezar algo nuevo" (p. 19). La cuestión política consiste entonces en "construir unos márgenes de maniobra suficientes, en las diferentes esferas que estructuran la existencia, para autorizar unas tomas de iniciativas y unas [realizaciones personales] llevaderas de un sentimiento auténtico de [consentimiento] a sí mismo en relación con el prójimo y el mundo, integrando tanto los requisitos de [la] dimensión social de la libertad como la relación a la realidad" (p. 19).

Pueden distinguirse cinco esferas en la capacidad de actuar no sustituibles unas por otras:

- La primera engloba las cuestiones industriales y laborales, “indisociables de la fábrica de los bienes y servicios que responden a unas necesidades y deseos" (p.21).

- La segunda es "la del poder adquisitivo y de las condiciones de vida, a la vez condición del desarrollo [personal] de cada individuo y [de la] capacidad de actuar específica" (p.22).

- La tercera alude a las esferas personales y familiares, "que no son solamente unos estados necesarios, sino también unas maneras de actuar" (p.22). 
- La cuarta pone énfasis en la relación al mundo, "empezando por el conocimiento y la imaginación" (p.22).

- La quinta es la de la acción común, "la esfera decisiva de la libertad política" (p.22).

En el segundo capítulo, que se interesa por la capacidad de actuar produciendo, Wisnia-Weill recuerda que "el trabajo remunerado organiza buena parte de nuestras vidas adultas", incluso cuando es incierto e inestable (p. 23). El lugar eminente del trabajo es una herencia del siglo XVIII, ya que "la modernidad ha valorizado al extremo las actividades productivas, comparativamente a la vida contemplativa y a la acción ciudadana" (p. 23). Pero, numerosas mutaciones acontecidas conducen a repensar las nuevas funciones del trabajo productivo (p. 23).

La primera función de la actividad económica hace referencia a la capacidad de emprender, producir y crear (p. 24). Además de representar la manera principal de ganarse la vida, "el trabajo afilia socialmente", abriendo el camino a la "ciudadanía social" (p. 24). Como lo indica Robert Castel, "es porque casi todo el mundo trabaja, y trabaja en el marco de un estatus al que están vinculados unas protecciones y unos derechos [consolidados], que prácticamente todo el mundo goza de cierta ciudadanía social" (Castel, 2008, p. 136). Pero, "el trabajo se ha convertido en incierto, marcado por unos cambios de estatus (...) y unas transiciones inestables entre empleo y no-empleo (...). Los jóvenes o los que no están cualificados son sus principales víctimas" (p. 27). Además, las revoluciones tecnológicas mundiales provocan "unos fenómenos de destrucción creativa de las empresas. (...) Estas mutaciones son fuente de trayectorias profesionales más entrecortadas" (p. 27).

Si tener un empleo productivo es una necesidad, "para que los individuos lo vivan como una [actividad, es necesario que] crean en [su seno un espacio de] participación reconocido [como] una actividad socialmente vital $\mathrm{y}$, si es posible, socialmente valorizante" (p. 31). No en vano, "la transición ecológica obliga a cuestionar el estatus de la producción económica como bien común” (p. 31). Por lo cual, "un compromiso general de transformación de los modelos de negocios de las empresas en una perspectiva de neutralidad carbono acelerado (...) se impone, para que el trabajo siga siendo [una capacidad] de actuar, en el sentido de consentimiento a la participación de una producción como bien común" (p. 32).

Para la autora, "un sistema productivo en el cual un máximo de individuos consiente sin tristeza, e incluso con alegría, a [cumplir] los requisitos de la división del trabajo sería un avance social que aumentaría la [capacidad de actuar] de cada uno. Es la apuesta que parece hacer el capitalismo creativo" (pp. 34-35). En Europa, el 37\% de las personas trabajan en unas organizaciones aprendedoras consideradas como propicias para el desarrollo personal de los asalariados (p. 35). 
El problema es que la difusión de la automatización, de la robotización y de la inteligencia artificial favorece una polarización del mercado laboral "entre empleos a fuerte valor añadido, con gran margen de maniobra [para la] actividad individual, y empleos poco cualificados" y altamente subordinados (p. 35). Además, el fomento del entusiasmo puede ser un motor perverso fuente de sufrimiento en el trabajo, ya que el llamamiento a la realización personal a través de la actividad laboral es esencialmente externo al individuo (p. 36). A su vez, el imperativo permanente de ser autónomo puede ser sinónimo de agobio y de estrés, "sobre todo cuando conduce a negar la realidad de la vida en [la] empresa", dado que "siempre hay un momento en el cual la ejecución de la acción necesita una obediencia" (p. 36). Ante semejante panorama, sería conveniente proceder al desarrollo de "unas formas de economía colaborativa orientadas hacia la producción de bienes comunes"; al "refuerzo de la participación de los asalariados"; al "desarrollo de las formas de [gestión compartida] o de asociación a la fijación de unos objetivos de trabajo y de calidad" (p. 36).

En el tercer capítulo, que aborda la cuestión del poder adquisitivo y del consumo, la autora indica que unas "condiciones de vida y [un] poder adquisitivo satisfactorios son las piedras angulares del consentimiento democrático en una sociedad ampliamente organizada por la promesa de la prosperidad material, pero donde los hogares modestos están de nuevo atenazados por [el gasto comprometido]. Estas prioridades concentran los retos clásicos que afrontan las políticas de redistribución desplegadas por [los] Estados de bienestar frente a las divisiones desigualitarias" (p. 41).

Si el consumo no es directamente una esfera de actividad, "condiciona unos funcionamientos que cada uno necesita para vivir y para realizarse [personalmente]: una esperanza de vida suficiente y [unas] libertades civiles y [unos] derechos que garantizan la integridad física, una alimentación que evita [pasar hambre], una vivienda segura [y digna], pero también una [atención médica] y una educación básica para [poder] participar en la vida social" (p. 42). Además de permite vivir, el consumo constituye una capacidad de actuar (p. 43). Asimismo, "en nuestras sociedades, donde la parte de la autoproducción es muy débil, el consumo [autorizado] por la renta disponible, no es solamente la satisfacción de una necesidad o un disfrute, sino también una manera de socializarse, de forjarse una identidad y de influir el sistema de preferencias colectivas" (p. 44).

En los países europeos, “ciertos bienes [aparecen] como esenciales, hasta el punto de [generar] unas regulaciones [del] mercado, de ser [proporcionados por los] servicios públicos o financiados por el Estado de bienestar. Se trata de garantizar, [en un principio], un acceso mínimo a estos bienes” (pp. 44-45). Así, en 1999, “el $90 \%$ de los europeos [estimaban] importante garantizar los bienes básicos a todos 
[los ciudadanos] (educación, sanidad, alimentación, vivienda, vestido)" (p. 45). Por ejemplo, si la alimentación es una necesidad primaria de todas las sociedades, en los países desarrollados, la población accede a la alimentación a través del mercado, "dado que los precios de estos bienes han [disminuido] suficientemente [como] para [representar] una escasa parte de los presupuestos de los hogares, incluso modestos" (p. 46). Pero, si la subalimentación crónica ha desaparecido, (...) el $0,9 \%$ de la población declara no tener suficiente comida a menudo o a veces" (p. 46).

Al contrario, "los bienes y servicios de sanidad están ampliamente [ofrecidos] por la potencia pública (investigación, hospitales, sanidad pública), aunque algunos [de ellos] estén proporcionados por el mercado (innovación farmacéutica, complementaria sanitaria, prácticas liberales)" (p. 46). Además, Francia ha desarrollado progresivamente una cobertura sanitaria universal para las personas enfermas ofreciendo una atención médica integral. "El seguro médico, la cobertura [médica] universal complementaria y el [reembolso] al 100\% de los cuidados y tratamientos vinculados a una [enfermedad de larga duración, representan] unas respuestas a las necesidades de los pacientes" (p. 47). No en vano, el debate sobre el umbral mínimo aceptable de funcionamiento de la sanidad no está agotado y alude a las desigualdades de acceso efectivo a la sanidad. A su vez, la financiación del sistema de atención a las personas que padecen enfermedades mentales es insuficiente (p. 47).

La educación es igualmente "un bien esencial ampliamente proporcionado por la potencia pública" (p. 48). Pero, cumple, ante todo, "un objetivo de igualdad simple para todos (zócalo común de conocimientos) (...) y de redistribución (educación prioritaria)" (p. 48). En ese sentido, no da una respuesta a cada uno según sus necesidades (p. 48). De hecho, "si se desea posicionar la educación como [un] bien esencial que responde a las necesidades específicas de cada [alumno] para alcanzar un nivel [mínimo] de conocimientos y de competencias, la [escuela] obligatoria está mal equipada. Cerca del $20 \%$ de los jóvenes de 16 a 24 años no tienen el nivel que les permite [participar] en la vida activa y social" (p. 48). Si el desdoble de las clases de primaria, la prioridad concedida a los aprendizajes fundamentales, las pedagogías eficaces o la mejora de las carreras de los profesores van en la buena dirección, no permiten solucionar el problema de los jóvenes en gran dificultad (p.48).

Por último, "la crisis ecológica podría [ofrecer] la oportunidad de dar un rol más importante a la orientación por los ciudadanos de una estructura de bienes que responda a sus aspiraciones" (p. 56). Asimismo, "para evitar deteriorar las condiciones de vida materiales, la descarbonización [del consumo más corriente] es indispensable, pero no es la única vía [posible]" (p. 56). Otras soluciones existen, 
tales como "reequilibrar el acceso al ocio, ordenando el territorio con unas utilidades de proximidad dedicadas, [elaboradas] según las normas ecológicas" (p. 56). Para luchar contra el desorden climático, unos instrumentos de intervención pública a largo plazo, complementarios de los del sector privado, podrían ser financiados por la creación monetaria, el fomento de las monedas locales, el aumento de la fiscalidad que pesa sobre las rentas más altas o la incentivación a convertirse en accionistas de empresas que aspiran a ser neutras en materia de emisiones de carbono (p. 57).

En el cuarto capítulo, que se interesa por la capacidad de amar y de practicar la reciprocidad, le autora observa que, si la familia reproduce, en la medida en que, en su seno, se educa a los hijos, se transmite el capital económico y cultural y se garantiza la satisfacción de las necesidades básicas, es también la primera esfera relacional. Esta esfera de los lazos íntimos aparece esencial para la capacidad de actuar (p. 60). No obstante, la familia puede estar amenazada por un régimen político (el totalitarismo o la dictadura que restringen las libertades), la estructura económica (la organización del trabajo y del territorio que determina ampliamente el tiempo dedicado a sus familiares) o la vida digital (el tiempo consagrado a Internet y a las redes sociales no para de crecer) (p. 62).

En efecto, "la seguridad procurada por unas figuras de apego estables es vital para los niños en su desarrollo, especialmente afectivo. Está admitido, hoy en día, que [incumbe] a un Estado capacitante garantizar [la protección] de los niños maltratados (...) en nombre de los derechos de los niños y de las responsabilidades de los padres" (p. 63). Para ello, es preciso hacer evolucionar los dispositivos de atención para responder mejor a las necesidades relacionales de los niños. (...). Los niños protegidos deben poder vivir unas situaciones de afecto aseguradoras forjando la posibilidad de vincularse [a los demás]" (p. 63). Cultivar la facultad de vincularse, que desemboca en una capacidad de actuar, implica recurrir a una atención psicoterapéutica que se caracteriza por una gran desigualdad de acceso.

Pero, las demás instituciones tienen un rol de prevención y de reparación, "especialmente ofreciendo a los niños y a los jóvenes [unas] experiencias más variadas que [permiten relacionarse] en pequeños grupos, aprender el gusto de la conversación amistosa en unas formas de intimidad [tranquilizantes]” (p. 64). Otros vectores de socialización amistosa pueden ser activados "utilizando las complementariedades de la sociedad digital y de los territorios", por ejemplo, favoreciendo las agrupaciones de amigos en la escuela, proponiendo unos proyectos fuera de la clase, etc. (p. 65). Estas políticas "permiten establecer unos vínculos fuertes, pero también unos vínculos más impersonales" (p. 65).

De la misma forma, uno no nace padre, sino que se convierte en ello "tejiendo unas capacidades que permiten al niño devenir sí mismo, [entrelazando] unas relaciones entre padres e hijos que presiden a la atmósfera emocional de [sus] vidas" 
(p. 66). Pero, existen ciertas condiciones objetivas que lo propician. En primer lugar, la capacidad "del padre para responder en el buen momento a las pruebas que atraviesan [sus hijos]" (p. 67). En segundo lugar, las que proporcionan el cuidado, generalmente las mujeres, deben poder trabajar en el exterior de sus hogares de manera remunerada (p. 67). Lo cierto es que "el auge de las parejas bi-activas o de las [familias] monoparentales, combinado con las mutaciones del trabajo, modifican las tensiones entre vida familiar y vida profesional" (p. 68).

Pero, concebir "unas políticas a favor de la conciliación vida familiar-vida profesional como una manera de activar el acceso de las mujeres al mercado laboral responde insuficientemente a los retos relacionales de la paternidad como [actividad]" (p. 69). Además de convertir las consecuencias del trabajo parental en equitativas, "convendría impulsar una política de la actividad parental, que tampoco sea una profesionalización de la parentalidad" (p.69). Esto implica descentrar "las políticas públicas de una [concentración] en la pequeña infancia" (p. 69).

A su vez, según la autora, convendría fomentar "un régimen de protección social que continué articulándose [en torno a] las solidaridades familiares, en lugar de sustituirse a ellas o de limitarse a ellas" (pp. 73-74). De hecho, "la reciprocidad comprometida es [una acción] intrínseca que [posibilita] la libertad social común. Convoca una ética de la relación al largo plazo y a la deuda" (p. 74). Y, "el reconocimiento mutuo de los lazos íntimos construye una parte de la relación a la realidad y a la alteridad, [que constituyen los] fundamentos del trabajo, del conocimiento y de la ciudadanía como [capacidad de actuar]" (p. 74).

En el quinto capítulo, que analiza la capacidad de conocer y de transformarse gracias al contacto con el mundo, la autora observa que llevar a cabo un pensamiento reflexivo, "en cuanto a la manera de llevar a cabo su vida, forjarse una concepción de la justicia, saber argumentar. El ejercicio de la razón práctica y el desarrollo del espíritu crítico están en el corazón de la libertad de pensar, decisiva para cualquier conocimiento" (p. 77). Piedra angular de la capacidad de gobernarse a sí mismo, poderoso vector de desarrollo de los derechos individuales, "esta subtiende igualmente una democracia deliberativa" (p. 77). Si todas las personas están capacitadas para pensar, "la razón práctica es una facultad que se cultiva [precozmente]" (p. 77). A ese respecto, conviene distinguir la necesidad de razonar y la exigencia de aprender a reflexionar con vivacidad (p. 78).

A su vez, es preciso conservar la capacidad de actuar del conocimiento humano (pp. 85-86). Esto implica "formular una estrategia [global relativa a los buenos niveles a alcanzar]" (p. 86). La elaboración de semejante estrategia representa un reto en materia de competitividad, ya que incide en la subida en gama de la estructura de empleo (p. 86). Esta estrategia no puede concernir solamente la enseñanza superior y tampoco puede abstraerse de la comparación internacional (p. 86). 
Elaborar" una estrategia de buenos niveles con unas trayectorias diversificadas (...) ampliaría la base de los buenos alumnos y subiría el nivel [durante] la escolaridad obligatoria. Esto engendraría una democratización del acceso a las excelencias entre los adolescentes" (p. 87). Esta pluralización y personalización de las esferas de realización personal desde la enseñanza secundaria permitiría "completar la lucha contra las desigualdades" (p. 87). Y ello por dos razones. Por una parte, la escuela debe tomar en consideración precozmente la necesidad de transgresión y de búsqueda de intensidad, ideal y construcción de una singularidad de los adolescentes "organizando unas posibilidades de superación [traducidas] en unos reconocimientos" (p. 88). Por otra parte, en las sociedades en las cuales el individuo debe realizarse a través de su actividad, "amplificar las posibilidades de realización [personal] supone [reconsiderar] la distribución de los honores" (p. 88). Por lo cual, conviene "pluralizar las vías de excelencia" (p. 88). Esto implica, en primer lugar, detenerse en la enseñanza profesional y tecnológica secundaria (p.88). Supone, en segundo lugar, interesarse por el labelling a través de la enseñanza superior y de los concursos de acceso a la función pública" (p. 89). A su vez, "unas desigualdades de renta más limitadas y repartidas en [un mayor número] de funciones convertirían en más realista y aceptable esta pluralización de las esferas de excelencia" (p. 90).

En el sexto capítulo, dedicado a la democracia o a la capacidad de actuar en común, Wisnia-Weill indica que las cuatro precedentes esferas referidas a la capacidad de actuar (producción, poder adquisitivo, capacidad de amar y capacidad de conocer) "dan cuenta de [unas capacidades de actuar] fuera de la política" (p. 92). No en vano, construir un mundo común sigue siendo una capacidad de actuar que el movimiento de los Chalecos Amarillos aparecido en Francia en 2019 ha convocado en varias ocasiones (p. 92). Por un lado, "la necesidad de compartir lo común que [alude] a lo que nos une" (p. 92). Por otro lado, la exigencia de participar efectivamente en las elecciones políticas (p. 92). Ambas confluyen para edificar una sociedad en términos sociales y políticos (p. 92).

La insatisfacción ante una ciudadanía demasiado puntual, "mientras que la masificación escolar y la sociedad digital producen un mundo donde cada uno puede [expresar] públicamente su opinión y desea ser escuchado, parece deber desembocar en unas deliberaciones más numerosas, susceptibles de escandir regularmente la vida democrática. Los referendos de iniciativa ciudadana y de iniciativa compartida son sus encarnaciones" (p. 94). Para evitar la descalificación de los ciudadanos y la subordinación a las opiniones públicas, es preciso que "los ciudadanos (...) que desean participar más regularmente sean puestos en posición de ser unos productores de conocimiento" (p. 95). Se trata de movilizar, "en el proceso [de elaboración], el saber vivencial que cada uno posee (...) para formular una recomendación de interés 
general. Es también la vía para que la participación ciudadana no sea solamente la respuesta a la demanda de ser escuchado" (p. 96).

Multiplicar los dispositivos de participación puede ser virtuoso. Por ejemplo, incluyendo la sociedad civil en el trabajo de la comisión del Parlamento, creando jurados ciudadanos, haciendo evolucionar el Consejo económico, social y medioambiental o creando unos comités ciudadanos encargados de acompañar unos trabajos de evaluación de las políticas públicas (p. 96). A ese respecto, la revolución digital ofrece nuevas medidas y nuevas apetencias; por ejemplo, en el ámbito sanitario con la creación de grupos de discusión o de foros de pacientes (p. 96).

La ciudadanía de producción de conocimiento "permite ampliar considerablemente el juego rio arriba de la elaboración de las políticas públicas" (p. 97). Pero, dado que el Estado democrático está lejos de tener el monopolio de la acción, "al lado de lo que depende directamente de la responsabilidad operativa (...), busca impulsar unas dinámicas ante unos ciudadanos y unas empresas en [colaboración] con las [administraciones] locales" (p.98). Así, “el Estado podría ampliamente desarrollar su rol de arquitecto [aglutinador] para asociar las diferentes acciones en el tiempo y hacer emerger unas soluciones localmente pertinentes y socialmente justas" (p. 100).

En el apartado de conclusiones, donde la autora aspira a democratizar las capacidades de actividad, observa que, "más allá de las transformaciones empresariales inherentes a cualquier revolución tecnológica, es necesario democratizar [dichas capacidades]. Distinguir la actuación [constructiva] y el activismo estéril o una eficacia [poco enraizada] y acordarse sobre lo insustituible (...) aparece necesario" (p. 105). De hecho, "del corazón de una libertad de actuar socialmente benéfico, pensado como unas coacciones de la alteridad y el sentido de las realidades afectivas, nacen los retos de justicia social que la urgencia climática convierte en indispensables" (p. 108).

Al término de la lectura de Les nouveaux pouvoirs d'agir, es preciso subrayar la gran actualidad y pertinencia de la reflexión desarrollada por la autora sobre las nuevas capacidades de actuar en un mundo globalizado en plena mutación. Esta reflexión, que se basa en la teoría de las capacidades, se nutre de numerosas lecturas y de los innumerables informes redactados por Wisnia-Weill, en solitario o en colaboración con otros autores, sobre las políticas sociales y educativas. Las tesis expuestas, a pesar de su alto nivel de abstracción, están ilustradas por varios casos concretos y cuadros temáticos, lo que confiere solidez analítica y fundamentación empírica a la obra. Asimismo, no duda en formular propuestas que aspiran a incrementar las capacidades de actuar de las personas en los diferentes ámbitos. No en vano, a veces, el lector tiene la sensación de que la autora realiza un mero repaso de la literatura filosófica, poniendo especial énfasis en las obras de Amartya Sen (1985) y Martha Nussbaum (2011).

En cualquier caso, la lectura de esta obra es sumamente estimulante para identificar y analizar las nuevas capacidades de actuar. 


\section{BIBLIOGRAFÍA}

Castel, R. (2008): “La citoyenneté sociale menacée”, Cités, vol. 3, 35, pp. 133-141.

De Singly, F. y Wisnia-Weill, V. (2015): Pour un développement complet de l'enfant et de l'adolescent. París: France Stratégie.

(https://www.strategie.gouv.fr/sites/strategie.gouv.fr/files/atoms/files/fs_rapport commission_enfance 29092015 bat.pdf).

Jolly, C., Prouet, E. y Wisnia-Weill, V. (2016): Repenser la protection des actifs. París: France Stratégie. (https://www.strategie.gouv.fr/sites/strategie.gouv.fr/files/atoms/files/2017-2027actions-critiques-protection-actifs.pdf).

Jolly, C., Prouet, E. y Wisnia-Weill, V. (2017): Comment mieux protéger les actifs du risque de perte ou d'insuffisance de revenus? París: France Stratégie. (Disponible en: https://www. strategie.gouv.fr/debats/mieux-proteger-actifs-risque-de-perte-dinsuffisance-de-revenus).

La vie des idees (2019): Wisnia-Weill Vanessa. (https://laviedesidees.fr/ Wisnia-WeillVanessa .html).

Naussbaum, M. (2011): Capabilités. Comment créer les conditions d'un monde plus juste? París: Climats.

Sen, A. (1985): Commodities and Capabilities. Amsterdam: North-Holland.

Walzer, M. (1983): Sphères de justice. Paris: Seuil.

Wisnia-Weill, V. (2014): Augmenter aussi le nombre de bons élèves. París: France Stratégie. Disponible en: https://www.strategie.gouv.fr/sites/strategie.gouv.fr/files/atoms/files/201405-20-pisa-ok1.pdf

Wisnia-Weill, V. (2018): “Aménagements souples du temps et du lieu de travail”, in Disposer de temps et de droits pour s'occuper de ses enfants, de sa famille et de ses proches en perte d'autonomie. París: HCFEA. 